ISSN 1807-975X

\title{
O sentimento de inveja suscitado pelo consumo de produtos turísticos divulgado no Instagram
}

The feeling of envy aroused by travel consumption posted in Instagram

\section{El sentimiento de envidia en el consumo de viajes a partir del contenido visualizado en Instagram}

\author{
Elnivan Moreira de Souza ${ }^{1}$ \\ Rebeca Maria Bruno Montenegro² \\ Izabelle Quezado ${ }^{3}$ \\ Danielle Miranda de Oliveira Arruda ${ }^{4}$
}

\begin{abstract}
Resumo: Este artigo verifica o papel do sentimento de inveja no consumo de viagens a partir do conteúdo visualizado no Instagram. Para tanto, foi realizada uma pesquisa quantitativa por meio de survey e os dados foram tratados com auxílio dos softwares SPSS, para análise descritiva e validação das escalas, e AMOS, para análise das relações regressoras entre as variáveis observáveis e os construtos. Constatou-se que os integrantes da amostra da pesquisa são potenciais consumidores de viagens, pois as realizam e acessam o Instagram com frequência, mas não são pessoas que tem o sentimento de inveja como determinante. Conclui-se afirmando que a mídia social Instagram não suscita o sentimento de inveja dos seus usuários no que se refere ao consumo de viagens. Como contribuição, ressalte-se a validação das escala de Inveja e Identificação Social que ainda não haviam sido validadas no Brasil. Para estudos futuros, propõe-se verificar a influência do sentimento por meio de outras mídias sociais como Facebook e Twitter, além de outros nichos de mercado relevantes. Metodologicamente, indica-se o uso de abordagens qualitativas, apoiada por técnica como análise do discurso, pois essa possibilitará uma análise minuciosa, capaz de detectar lacunas não cobertas pela abordagem quantitativa.
\end{abstract}

Palavras chave: Inveja; Consumo de viagens; Instagram.

Abstract: This article aims at investigating the role of envy in travel consumption from content viewed on Instagram. To this purpose, a survey was carried out and the dada was analyzed using SPSS software, for descriptive analysis and validation of the scales, and AMOS, to analyze the regressive relations between the observable variables and the constructs. It was found that the members of the research sample are potential consumers of travel, because they carry out and access Instagram frequently, but they are not people who have the envying feelings as determinant. It is concluded that the social media Instagram does not give rise to the envy of its users when it comes to travel consumption. Furthermore, as contribution, we highlight the validation of the Envy and Social Identification scale that had not yet been validated in Brazil. For future studies, it is proposed to verify the influence of feeling through other social media such as Facebook, Twitter and other relevant market niches. Methodologically, the use of qualitative approaches is indicated, supported by technique such as discourse analysis, as this will allow a detailed analysis, capable of detecting gaps not covered by quantitative method.

Keywords: Envy; Travel consumption; Instagram.

\footnotetext{
${ }^{1}$ Doutorando em Administração pela Universidade de Fortaleza (UNIFOR). Professor e integrante do Núcleo Docente Estruturante (NDE) do curso de Administração do Centro Universitário Christus (UNICHRISTUS). Email: elnivan@hotmail.com.

${ }^{2}$ Mestre em Administração de Empresas pela Universidade de Fortaleza (UNIFOR). E-mail: rebecamontenegro71@gmail.com.

${ }^{3}$ Doutora em Administração de Empresas pela Universidade de Fortaleza (UNIFOR). E-mail: izabellequezado@gmail.com.

${ }^{4}$ Doutora em Administração de Empresas pelo Institut d'Administration d'Entreprises de 1'Université de Nice França. Professora Associada da Universidade Estadual do Ceará (UECE). E-mail: danielle.gomes@uece.br.
} 
Resumen: Este artículo investiga el papel del sentimiento de envidia en el consumo de viajes a partir del contenido visualizado en Instagram. Para ello, se realizó una investigación cuantitativa a través de survey y los datos fueron tratados con ayuda de los softwares SPSS, para análisis descriptivo y validación de las escalas, y AMOS, para análisis de las relaciones regresivas entre las variables observables y los constructos. Se constató que los integrantes de la muestra de la investigación son potenciales consumidores de viajes, pues las realizan y acceden al Instagram con frecuencia, pero no son personas que tienen el sentimiento de envidia como determinante. Se concluye afirmando que los medios sociales Instagram no suscita el sentimiento de envidia de sus usuarios en lo que se refiere al consumo de viajes. Como contribución, se resalta la validación de las escalas de Envidia e Identificación Social que aún no habían sido validadas en Brasil. Para estudios futuros, se propone verificar la influencia del sentimiento por medio de otros medios sociales como Facebook y Twitter, además de otros nichos de mercado relevantes. Metodológicamente, se indica el uso de enfoques cualitativos, apoyada por técnica como análisis del discurso, pues ésta posibilitar un análisis minucioso, capaz de detectar lagunas no cubiertas por el abordaje cuantitativo.

Palabras clave: Endivia; Consumo de viajes; Instagram.

\section{INTRODUÇÃO}

Frutos da Revolução Industrial, a oferta de bens em massa e a comunicação voltada para a simbologia do consumo, geraram, na classe média, grande demanda por produtos antes restritos às camadas sociais mais abastadas. Esse consumidor emergente passou a desejar o status social dos indivíduos pertencentes ao topo da pirâmide econômica, realidade que elevou padrões de consumo e alavancou o desenvolvimento da economia (TUROW, 2006).

O comportamento de compra motivado pelo desejo de consumir produtos previamente adquiridos por pessoas ou grupos de referência é, atualmente, enfoque de artigos na seara de Marketing (e.g. BELK, 2008; BELK, 2011; FOLKES, 2012; FOTIS; BUHALIS; ROSIDES, 2012; KONG, 2015), que visam a explorar a emoção de inveja enquanto antecedente da conduta humana (BELK, 2008).

Embora o tópico venha sendo explorado recentemente, pesquisas adicionais são necessárias no intuito de melhor compreender esses mecanismos cognitivos inerentes a escolhas de consumo, uma vez que, frente à numerosa oferta de produtos do mercado contemporâneo, consumidores têm uma maior tendência a considerar seus estados emocionais, como o de inveja, em processos decisórios de compra (FOLKES, 2011). Ainda, Belk (2011) reforça a importância dessa temática ao afirmar que o comportamento do consumidor motivado pela emoção de inveja merece mais atenção de pesquisadores no campo do Marketing.

$\mathrm{Na}$ presente pesquisa, investiga-se a inveja à luz da estrutura estímulo-organismoresposta (E-O-R), que tem, por base, o sistema cognitivo por meio do qual o indivíduo processa características do ambiente e interpreta situações, atribuindo-as algum significado (LAZARUS, 
1991; PETER; OLSON, 1994). Especificamente, neste estudo, explora-se, como estímulo, o conteúdo associado ao consumo de viagens, publicado em ambiente virtual, e a possível emoção de inveja, decorrente do julgamento cognitivo desse conteúdo por quem o visualiza.

A relevância em explorar o meio digital é apresentada no estudo de Belk (2011), que traz indícios de que este ambiente é uma lacuna na teoria em torno de inveja e consumo. Segundo o autor, uma vez que permite que o indivíduo acompanhe, de forma remota, mas assídua, o que outros consomem, a internet fomenta a identificação social. De acordo com ensaios no âmbito da psicologia (ROSA, 2005), essa identificação está intimamente ligada à emoção de inveja, que pode despertar o desejo de copiar condutas de consumo de pessoas ou grupos de referência.

Assim, com a disseminação das plataformas sociais no ambiente virtual, atribui-se, ao comportamento do consumidor, novos estímulos que motivam o consumo (AYEH; AU; LAW, 2013). No entanto, embora estudos atestem a influência do conteúdo virtual em decisões de compra, com destaque ao exposto em redes sociais (FOTIS; BUHALIS; ROSSIDES, 2012), não foram encontradas pesquisas que explorassem reações ao sentimento de inveja frente à divulgação, em plataformas sociais, de produtos adquiridos por outros consumidores.

Reconhecendo essa lacuna teórica, este estudo objetiva verificar o papel do sentimento de inveja no consumo de viagens a partir do conteúdo visualizado no Instagram, rede social de compartilhamento de fotos detentora de vasta popularidade e de intensa utilização entre os usuários (KONG, 2015).

De acordo com Kong (2015) o conceito de uso do Instagram baseia-se na publicação de fotos que retratam a realidade do dia a dia do usuário, divergindo, assim, de outras redes sociais, que se constituem pela postagem de fotos mais selecionadas, editadas e menos representativas do real cotidiano do utilizador. Desse modo, acredita-se que o atributo de veracidade inerente ao conteúdo dessa rede social, em comparação às demais, é mais favorável a suscitar a emoção de inveja mediante fotos que expõem o consumo de produtos por outros usuários.

Especificamente no que diz respeito ao consumo de viagens turísticas, este vem se destacando, em pesquisas, por sua grande ascensão em decorrência da facilidade de busca por informações no ambiente virtual (FOTIS; BUHALIS; ROSIDES, 2012). De acordo com o World Travel Market (2010), um em cada três consumidores de pacotes turísticos faz uso de 
algum tipo de rede social online durante o processo de aquisição desse serviço. Nessa perspectiva, redes sociais, como o Instagram, que possibilitam a divulgação de experiências de viagens pelos usuários por meio de fotografias, podem vir a despertar o desejo de cópia do comportamento de consumo (KONG, 2015).

No entanto, vale ressaltar que a representatividade do Instagram no meio virtual não se reflete na seara acadêmica, uma vez que a maioria dos estudos que abordam redes sociais dedicam-se a explorar o Facebook (VISENTINI; BOBSIN; CHAGAS, 2015), o que também se configura como relevância do presente estudo.

Com base nos argumentos supracitados e visando a alcançar o objetivo proposto, optouse por desenvolver uma pesquisa quantitativa por meio de survey, composta pelas escalas de Belk (1984; 1985) para mensurar a inveja e de Bettman (2005) para avaliar a identificação social, além de questões em torno da frequência de viagens e acesso ao Instagram. A análise dos dados foi realizada com o auxílio do SPSS, para análise descritiva e validação das escalas, e do AMOS, para análise das relações regressoras entre as variáveis observáveis e os construtos.

Para embasar a pesquisa empírica, em seguida a esta introdução, o presente trabalho apresenta uma revisão teórica, que aborda identificação social e apresenta considerações em torno de consumo e inveja. Posteriormente, são apresentadas metodologia, análise dos resultados, conclusão e referências bibliográficas.

\section{REFERENCIAL TEÓRICO}

\subsection{Identificação Social}

Ao relacionar a sociedade contemporânea ao consumo, à abundância e à progressiva disseminação de bens e serviços, Baudrillard (2005, p. 95) observa uma sociedade na qual "o consumo surge como conduta ativa e coletiva", de modo que o grau com que o indivíduo se identifica com diferentes grupos sociais pode afetar seu comportamento no que diz respeito ao ato de consumir (ALGESHEIMER; DHOLAKIA; HERRMANN, 2005; BAGOZZI; DHOLAKIA, 2006). Há pesquisas que demonstram, como exemplo consequente da identificação social, o consumo de determinados produtos e serviços. Caso haja a identificação 
do consumidor com outros membros que aderem à marca, isso irá favorecer o comportamento de compra (DHOLAKIA; BAGOZZI; PEARO, 2004).

Identificação de consumidores com os outros pode, portanto, exercer forte influência em hábitos de consumo (DHOLAKIA et al., 2004; BAGOZZI; DHOLAKIA, 2006). No entanto, no âmbito do Marketing, ainda são restritas as pesquisas que se dedicam a explorar a identificação do consumidor com outros consumidores, por meio da aquisição, por parte destes, de bens ou serviços (KONG, 2015), sendo mais investigada a identificação com celebridades ou ícones sociais patrocinados por marcas (ESCALA, 2004).

A teoria que abrange a identificação social propõe que os indivíduos se organizam em diversas categorias sociais com o propósito de facilitar sua autodefinição perante o ambiente no qual está inserido. No geral, esta identificação é composta por diversas características humanas percebidas que moldam, primeiro, a identificação individual para, em seguida, formar a identificação social.

Esse sentimento está relacionado ao grau em que o indivíduo se sente parte de uma comunidade, enfatizando que a identificação social vai além da auto categorização percebida passando pela avaliação e estados afetivos com determinado grupo (TAJFEL; TURNER, 1985; ASHFORTH; MAEL, 1989; ASHFORTH; MAEL, 1989; ASHFORTH, 1992; BHATTACHARYA; RAO; GLYNN, 1995; STETS; BURKE, 2000; DHOLAKIA et al., 2004; ESCALAS, 2004).

Estudos prévios indicam que o principal aspecto da identificação social é o apego emocional (MCMILLAN, CHAVIS, 1986; ELLEMERS et al., 1999; BERGAMI; BAGOZZI, 2000). De acordo com Rosa (2005) a identificação está intimamente relacionada ao estado emocional de inveja, que pode despertar o desejo de copiar condutas de consumo de pessoas ou grupos de referência. A emoção de inveja, mediante o consumo de produtos por outros consumidores será abordada no tópico que segue.

\subsection{Inveja}

Até o final da década de 70, as pesquisas em marketing detinham-se a explorar o comportamento de compra racional do consumidor, ainda baseado no campo teórico da economia (FEHSE, 2009). Somente a partir da década de 80, com destaque ao estudo de Holbrook e Hirschman (1982), as emoções passaram a ser consideradas enquanto variáveis 
influenciadoras da decisão de compra. Embora a investigação desse substrato seja recente em Marketing, na literatura em geral, o conceito de emoções vem sendo explorado há muitas décadas nos mais diversos campos teóricos, incluindo a filosofia, por Aristóteles, Descartes e Spinoza (SANTOS; PRADO, 2008).

No que tange ao aspecto comportamental, Izard (1980), afirma que as emoções exercem influência direta nas percepções do ser humano, afetando, dessa forma, as ações decorrentes desse processo. Para Oliver (1997), o fato de a emoção ser resposta inerente às atividades do ser humano, as experiências de consumo também compõem os processos emotivos, uma vez que os indivíduos reagem a estímulos de consumo, de maneira positiva ou negativa. Em concordância, Richins (1997) aponta a inveja como uma das emoções mais recorrentes no comportamento do consumidor.

No esteio teórico das pesquisas de conduta do consumidor, o construto emoções é conceituado como uma resposta afetiva que resulta de uma experiência memorável do processo de consumo (HOLBROOK, 2000). Especificamente, a inveja é definida como uma emoção negativa que ocorre quando um indivíduo deseja algo que outro possui (HASMILOVIC; DINGUS, 2014).

Sob mesma perspectiva, Klein (1929) conceitua a inveja como um sentimento doentio, que experimenta-se quando é percebido o desejo pelo que o outro possui, incluindo a vontade de apropriar-se dele ou destruí-lo. Ademais, o sentimento de inveja é impulsionado por sua onipresença que passa para atos concretos logo que os indivíduos tornam-se capazes de se compararem, podendo render competição, hostilidade, alienação do sujeito que sente a inveja e o invejado (SCHOECK, 1969).

Pode-se inferir que não há somente o lado ruim e sombrio do sentimento, afirmativas emergem que a inveja também tem seu lado bom como o de motivar a economia, o de impulsionar esforços das pessoas a fim de trabalharem mais e com qualidade (ARISTÓTELES, 1954; EPSTEIN, 2003; KANT, 1780-1997; NEU, 1980).

Mais recentemente, Belk (2008) tem retratado inveja como algo positivo quando se refere, como por exemplo, a inveja entre os consumidores. Em pesquisa realizada em países europeus, aferiu-se que o sentimento pode ter caráter benigno ou maligno a depender de experiências, sentimentos, ideais e tendências de ação do indivíduo (ROSEMAN; WIEST; SWARTZ, 1994). 
Sobre essas duas perspectivas, para Hasmilovic e Dingus (2014) a inveja benigna, também conhecida popularmente por "inveja branca", pode levar à motivação. Neste sentido, o sentimento é abordado como um desejo construtivo para adquirir o bem cobiçado e obtê-lo. A inveja maligna, por sua vez baseia-se em uma sensação negativa por parte de um indivíduo, que acaba por desejar o retrocesso de quem inveja para sentir-se superior. Hellén e Saaksjarvi (2013) alertam para a teoria de que a "inveja branca" pode tornar-se maligna quando o consumidor se preocupa com seu status social em comparação aos demais consumidores.

Considerando o construto inveja como um todo, sem fazer menção a características positivas ou negativas, ora, a disseminação das plataformas de relacionamento online e o hábito social de propagar experiências de compra trazem novas perspectivas no que tange à inveja e consumo (BELK, 2011). Em concordância, Kong (2015) ressalta a necessidade de melhor conhecer a influência de consumo entre usuários comuns - não celebridades - de redes sociais.

\subsection{Mídias Virtuais}

Plataformas virtuais sociais vêm obtendo destaque no âmbito acadêmico internacional, porém, no que tange ao cenário de pesquisas brasileiras, ainda, não há compreensão clara em torno desse objeto, uma vez constituído por opiniões divergentes (VISENTINI; BOBSIN; CHAGAS, 2015).

As redes sociais fazem parte do dia a dia da sociedade intervindo em diversos aspectos e cenários no cotidiano das pessoas. Para Recuero (2009), as redes sociais são compostas de atores e conexões cujos atores são pessoas, grupos e instituições, também denominados de "nós" da rede e conexões são compostas pelos laços sociais e a interação entre os autores envolvidos.

No entanto, no que diz respeito a rede social virtual, Boyd e Ellison (2007) colocam que websites e aplicativos de redes sociais se definem por serviços na internet que possibilitam a criação de perfis individuais públicos ou semi-públicos em uma rede interligada a vários perfis, cujos usuários decidem como e com quem irão compartilhar informações possibilitando a visualização de outros usuários por meio da conexão com seus contatos dentro do sistema interligado que é a rede social virtual.

Raacke e Bonds-Raacke (2008) complementam relatando que além da comunicação com outros contatos, as redes sociais também fornecem flexibilidade midiática aos usuários, 
uma vez que estes podem divulgar informações sobre seus interesses através da publicação de textos, fotos e vídeos.

Em relação à forma de uso, Moraes et al. (2014) revelam que o uso das redes sociais se subdivide na categoria instrumental e sobritual. O uso instrumental está focado na busca de informações específicas, já o uso sobritual refere-se ao uso habitual como distração e entretenimento. No entanto, Fialho e Lutz (2011) citam alguns exemplos de websites como Facebook, Orkut, Linkedin, Twitter.

No que diz respeito ao estado da arte do tema, alguns dos trabalhos recentes que o compõe abordam os aspectos como: comunidades virtuais como estruturas de socialização (SCHRÖEDER; BERGER; KLERING, 2007), razões de aderência dos usuários às redes (DENG; TAVARES, 2013), dinâmica de ações coletivas estabelecidas em redes sociais virtuais (TAVARES; PAULA, 2013), redes sociais virtuais como meio de negócios (ARAÚJO; ZILBER, 2014) e marketing político por meio de plataformas virtuais (AMARAL et al., 2014).

\subsection{Modelo Teórico-Empírico}

Como resultado das argumentações teóricas das seções antecedentes, apresenta-se o modelo teórico empírico (Figura 1) e as hipóteses que lhe são subjacentes:

Figura 1 - Modelo Teórico Empírico

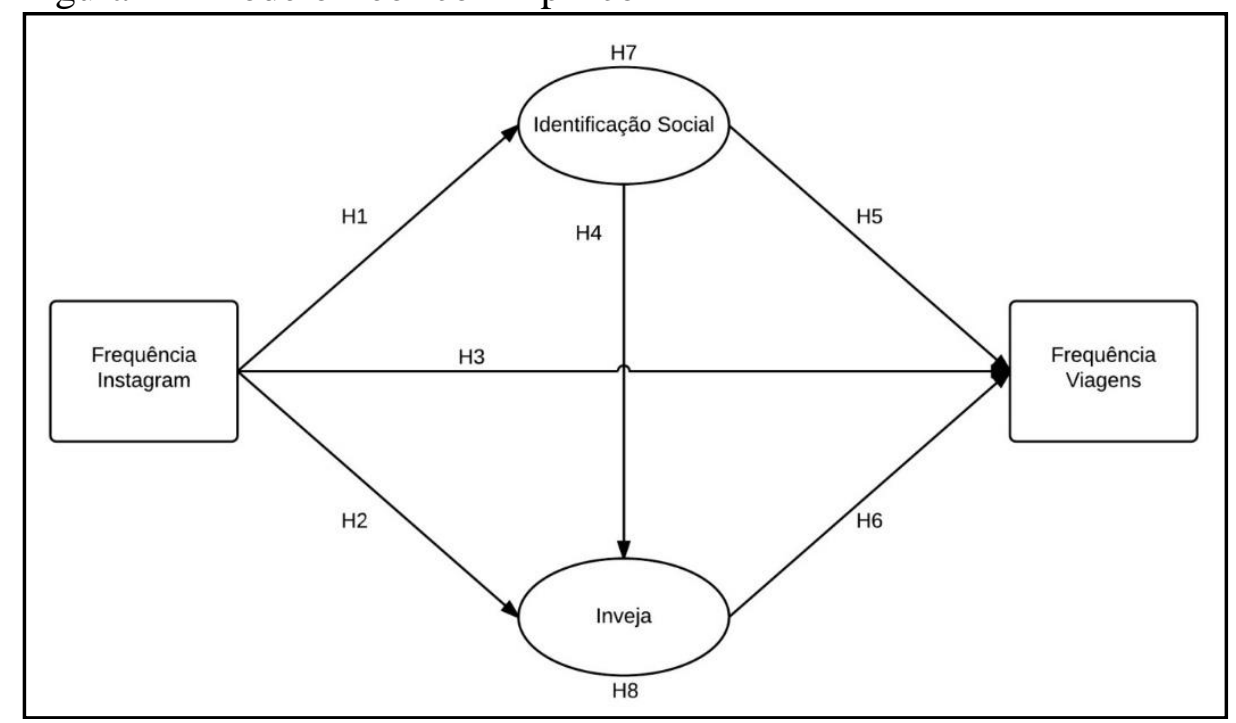

Fonte: Adaptado de Duff et al. (2012). 
H1 - A frequência de uso do Instagram (FI) influencia a Identificação Social (IS);

H2 - A frequência de uso do Instagram (FI) influencia a Inveja;

H3 - A Frequência de uso do Instagram (FI) influencia a frequência de Viagens (FV);

H4 - A Identificação Social (IS) influencia a Inveja;

H5 - A Identificação Social (IS) influencia a frequência de Viagens (FV);

H6 - A Inveja influencia a frequência de Viagens (FV).

H7 - A Identificação Social Media a relação entre a Frequência de Instagram e a Frequência de Viagens.

H8 - A Inveja media a relação entre a Frequência de Instagram e a Frequência de Viagens e entre Identificação Social e Frequência de Viagens.

\section{CAMINHO METODOLÓGICO}

\subsection{Caracterização da Amostra a Classificação da Pesquisa}

A presente pesquisa foi realizada junto a 202 usuários frequentes da mídia social Instagram. Escolheu-se esta mídia por ter como característica veicular imagens por meio de fotos ou vídeos, o que guarda aderência ao objetivo desta pesquisa. Um dos critérios de seleção dos respondentes consistiu em verificar se os usuários possuíam algum nível de identificação com pessoas que costumam viajar a lazer. Os dados foram coletados no mês de junho de 2015 com usuários do Instagram por meio de questionários eletrônicos. Quanto ao objetivo, a pesquisa é exploratória, pois os achados empíricos sobre o assunto ainda são exíguos. Em relação aos procedimentos, a estratégia de investigação combina pesquisa bibliográfica e survey. Os respondentes da pesquisa foram selecionados por técnica de amostra não probabilística por acessibilidade (BABBIE, 2001).

\subsection{Escalas Utilizadas e Instrumento de Pesquisa}

Foram usadas as escalas de Belk $(1984 ; 1985)$ para mensurar a Inveja e de Escalas e Bettman (2005) para avaliar a Identificação Social. As escalas foram inicialmente traduzidas, e em seguida, passaram por tradução reversa de um especialista (COSTA, 2011). Foram utilizadas variáveis qualitativas do tipo Likert de sete pontos.

$\mathrm{O}$ instrumento aplicado foi composto por questões relacionadas à frequência do uso da mídia social e do consumo de viagens, além das escalas já mencionadas. Para operacionalizar a 
escala de Identificação Social os autores optaram por fazer uso de imagens que remetiam as pessoas ao contexto de viagens de variadas formas (ex: lugares exóticos, inóspitos, pontos turísticos tradicionais, viagens a dois, em família ou em grupo). Os usuários também responderam questões relacionadas ao levantamento do perfil sócio demográfico, incorporadas à análise como variáveis de controle, sendo elas: profissão, nível de escolaridade, gênero, renda familiar e número de pessoas que dependem da renda.

Os itens das escalas foram submetidos a testes para avaliar a normalidade multivariada. Dado o tamanho da amostra, os testes usuais de ajustamentos à distribuição normal, como o Kolmogorov-Smirnov e Shapiro-Wilk, tornam-se sensíveis a pequenos desvios à normalidade, sendo desaconselháveis para aplicações como esse estudo (MARÔCO, 2010). Foram então avaliadas as medidas de assimetria e curtose e os maiores valores de coeficiente encontrados foram 1,567 e 2,139, respectivamente. Ambas as medidas se encontram dentro dos parâmetros descritos por Marôco (2010), valores inferiores a 2 para assimetria e inferiores a 3 para curtose, pressupondo a normalidade dos dados.

\subsection{Testes de Escalas e Métodos de Análise}

Em seguida, procedeu-se a validação das escalas e de suas dimensões, garantido a sua utilização. Para validação, utilizou-se a técnica estatística análise fatorial que busca, mediante avaliação de um conjunto de variáveis, identificar dimensões de variabilidade comuns existentes em um conjunto de fenômenos. Esta técnica objetiva simplificar ou reduzir um grande número de variáveis por meio da determinação das dimensões latentes comuns (fatores) (CORRAR et al., 2012; FÁVERO et al., 2009).

Os fatores extraídos foram submetidos ao teste de confiabilidade Alfa de Cronbach que mede a sua consistência interna, ou seja, indica se os itens individuais da escala realmente medem determinado constructo, estando altamente intercorrelacionados (CORRAR et al., 2012; FÁVERO et al., 2009). Após validadas as dimensões, foram testadas as relações conforme Modelo Teórico Empírico (Figura 1) (HAIR et al., 2009).

Além disso, foram também realizados testes para avaliar a existência de multicolinearidade entre as variáveis independentes do modelo de regressão, a fim de não comprometer os resultados das análises (Marôco, 2010). 
Para o tratamento dos dados foi utilizado o software Statistical Package for the Social Sciences (SPSS), módulos de estatística descritiva, análise fatorial e testes de confiabilidade de escala e o Analisys of Moment Structures (AMOS) para construção dos modelos de mensuração e estrutural. As técnicas de análise utilizadas foram: estatística descritiva; análise multivariada, compreendida por Análise Fatorial Confirmatória (AFC) e Modelagem de Equações Estruturais (MEE).

\section{ANÁLISE DOS DADOS}

\subsection{Análise Sócio Econômica e Descritiva dos Dados}

Uma caracterização inicial do perfil da amostra indica que a amostra teve uma participação maior de mulheres $(60,9 \%)$ em comparação aos homens $(39,1 \%)$. A maior parte dos respondentes tinham entre 21 e 30 anos $(68,8 \%)$, e no mínimo ensino superior completo $(60,9 \%)$. Em relação à renda 38,1\% informaram possuir uma renda familiar acima de R\$ 8.668,00 (dez salários mínimos), com uma média de 3,38 pessoas por família.

Sobre a frequência de acesso à mídia social Instagram, 38,1\% afirmaram acessar a mídia digital em intervalos inferiores a duas horas, 22,8\% afirmaram acessar a cada duas horas, 23,3\% indicaram acessar pelo menos uma vez por dia. Esses indicadores são condizentes com o que preconizam Raacke e Bonds-Raacke (2008) que relatam a relevância das redes sociais no papel da interação com outros contatos, além de também, fornecerem flexibilidade midiática aos usuários, uma vez que estes podem divulgar informações sobre seus interesses mediante publicação de textos, fotos e vídeos. Sendo assim, entende-se que o Instagram e seus recursos são ferramentas de grande valia para as empresas e sociedade quando utilizados de maneira estratégica. No que tange ao nível de frequência das viagens, 55\% afirmaram viajar pelo menos uma vez a cada semestre, 28,7 viajam uma vez por ano e 16,3\% viajam a cada dois anos.

Embora esse estudo não tenha como premissa básica a comparação entre gêneros no que se refere ao sentimento de inveja e à identificação social, optou-se por efetuar o teste $\mathrm{T}$ para verificar se não há diferença significativa entre as médias dos grupos. O teste T revelou que não há diferenças significativas no sentimento de inveja suscitado para nenhum dos dois grupos.

Utilizou-se a Correlação de Pearson para explorar os dados relativos à caracterização dos respondentes (TAB 1). Alguns achados mostraram-se coerentes com o senso comum, 
servindo como elemento para atestar a qualidade das informações coletadas. Em relação à Frequência de uso do Instagram (FI), esta apresentou correlação positiva significativa ao nível de 0,01 (TAB. 1) com idade e escolaridade (ESC). Sobre a Frequência de Viagens, esta apresenta correlação negativa apenas com a Renda. A Idade apresenta correção significativa com a FI, a Escolaridade, o sexo e a renda.

Tabela 1 - Correlações de Pearson de Análise Sócio-econômica e Descrição dos dados

\begin{tabular}{lrrrrrr}
\hline & FI & FV & IDADE & ESC & SEXO & RENDA \\
\hline FI & 1 & 0,046 & $0,332^{* *}$ & $0,259^{* *}$ & 0,000 & $-0,100$ \\
FV & 0,046 & 1 & $-0,022$ & $-0,106$ & 0,014 & $-0,176^{*}$ \\
IDADE & $0,332^{* *}$ & $-0,022$ & 1 & $0,353^{* *}$ & $0,170^{*}$ & $0,155^{*}$ \\
ESC & $0,259^{* *}$ & $-0,106$ & $0,353^{* *}$ & 1 & 0,125 & $0,315^{* *}$ \\
SEXO & 0,000 & 0,014 & $0,170^{*}$ & 0,125 & 1 & $0,144^{*}$ \\
RENDA & $-0,100$ & $-0,176^{*}$ & $0,155^{*}$ & $0,315^{* *}$ & $0,144^{*}$ & 1 \\
\hline
\end{tabular}

** A correlação é significativa ao nível de 0,01 (2 extremidades).

Fonte: dados da pesquisa.

De forma geral, segundo Tabela 2, a amostra se apresenta socialmente identificada aos grupos de pessoas que tem o hábito de viajar, pois apresentaram média superior a 3,5. Ainda segundo a mesma tabela, a amostra apresentou média inferior a 3,5 para o sentimento de inveja. Apresenta-se também os índices de assimetria e curtose para cumprir o critério de normalidade dos dados (MARÔCO, 2010).

Tabela 2 - Estatísticas Descritivas das Escalas de Inveja e Identificação Social

\begin{tabular}{l|r|r|r|r|r|r|r|r}
\hline & \multicolumn{1}{|c|}{$\mathrm{N}$} & \multicolumn{1}{c|}{ Média } & \multicolumn{1}{c|}{$\begin{array}{c}\text { Desvio } \\
\text { padrão }\end{array}$} & Variância & \multicolumn{2}{|c}{ Assimetria } & \multicolumn{2}{|c}{ Kurtosis } \\
\cline { 2 - 8 } & Estatística & Estatística & Estatística & Estatística & Estatística & $\begin{array}{c}\text { Erro } \\
\text { padrão }\end{array}$ & Estatística & $\begin{array}{c}\text { Erro } \\
\text { padrão }\end{array}$ \\
\hline IS1 & 202 & 4,60 & 1,888 & 3,564 & $-0,320$ & 0,171 & $-0,879$ & 0,341 \\
IS2 & 202 & 3,89 & 1,723 & 2,967 & 0,125 & 0,171 & $-0,796$ & 0,341 \\
IS3 & 202 & 4,12 & 1,775 & 3,150 & $-0,063$ & 0,171 & $-0,880$ & 0,341 \\
INV1 & 202 & 1,81 & 1,190 & 1,417 & 1,567 & 0,171 & 2,139 & 0,341 \\
INV2 & 202 & 2,29 & 1,894 & 3,589 & 1,363 & 0,171 & 0,647 & 0,341 \\
INV3 & 202 & 2,17 & 1,282 & 1,643 & 1,041 & 0,171 & 1,006 & 0,341 \\
INV4 & 202 & 3,38 & 1,776 & 3,152 & 0,254 & 0,171 & $-0,880$ & 0,341 \\
INV5 & 202 & 3,13 & 1,856 & 3,446 & 0,437 & 0,171 & $-0,966$ & 0,341 \\
INV6 & 202 & 2,03 & 1,414 & 1,999 & 1,504 & 0,171 & 1,925 & 0,341 \\
INV7 & 202 & 2,24 & 1,557 & 2,423 & 1,108 & 0,171 & 0,243 & 0,341 \\
INV8 & 202 & 2,76 & 1,854 & 3,438 & 0,875 & 0,171 & $-0,137$ & 0,341 \\
\hline FOnte: & & & & & & & &
\end{tabular}

Fonte: dados da pesquisa. 
Na Tabela 3 que segue, buscou-se responder em parte o objetivo do estudo. Foi feito um cruzamento entre a frequência que os respondentes viajam e a frequência que estes utilizam o Instagram. Dos que viajam numa frequência semestral, 10 respondentes afirmaram utilizar o Instagram a cada 5 minutos, 8 respondentes a cada 15 minutos, 8 respondentes a cada 30 minutos, 9 respondentes a cada hora e 20 respondentes a cada duas horas. Dos que viajam anualmente, 11 respondentes utilizam a ferramenta a cada hora e 24 respondentes a cada duas horas.

A estes resultados pode-se destacar os potenciais consumidores de viagens, uma vez que, viajar com essa frequência é relevante para o turismo, como também, ao fazer relação com a frequência que os mesmos usam o Instagram, percebe-se que a ferramenta é vantajosa para divulgar e promover o setor.

Segundo Sampaio et al (2008) o progresso do turismo, a complexidade que envolve suas atividades como fenômeno reivindicam um entendimento mais amplo considerado de uma forma igualitária sob aspectos sociais, econômicos e do ambiente. Sendo assim, é imprescindível debater a importância do consumo para atividades que envolvem o turismo.

Tabela 3 - Cruzamento entre Frequência de uso do Instagram e Viagens

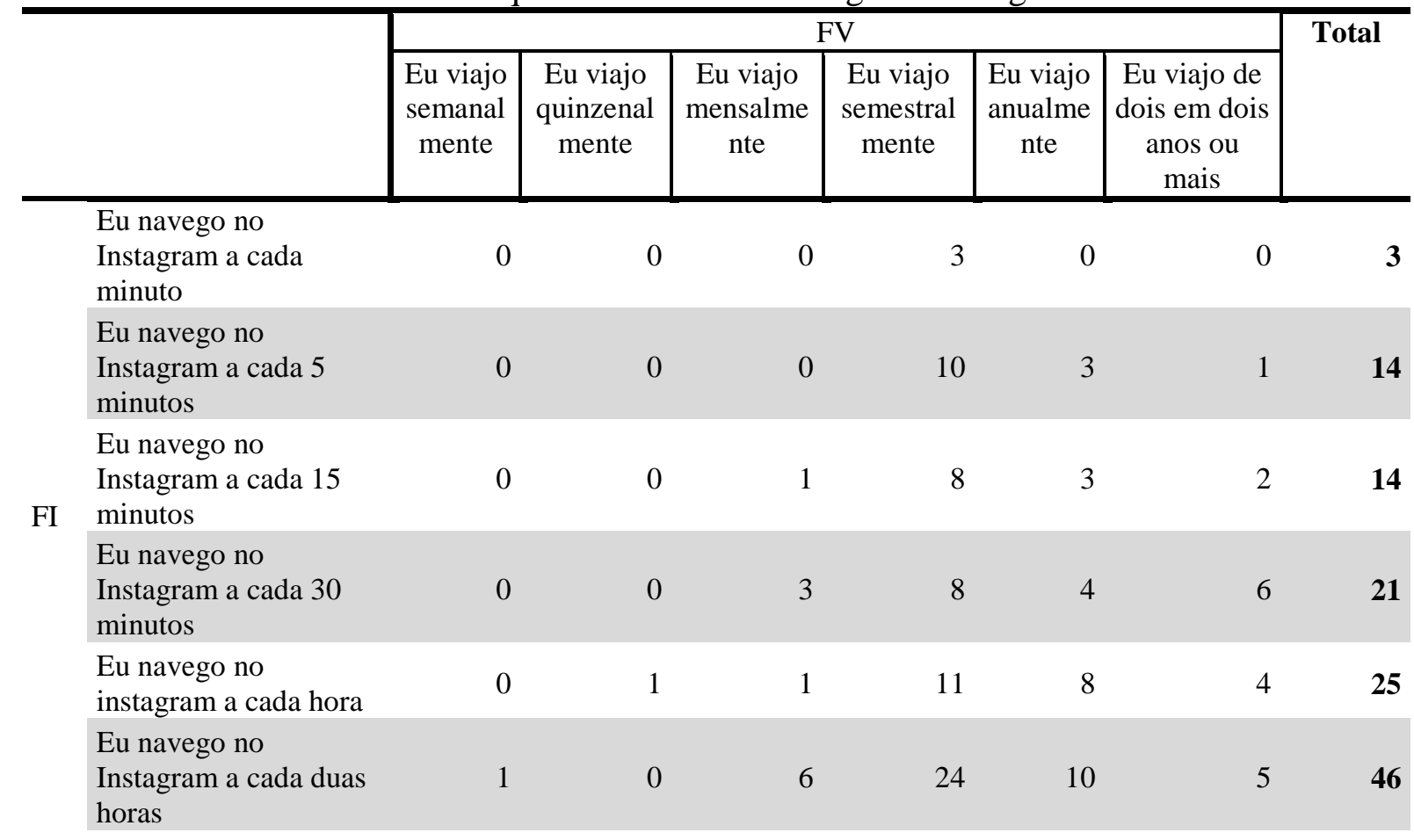




\begin{tabular}{|c|c|c|c|c|c|c|c|}
\hline $\begin{array}{l}\text { Eu navego no } \\
\text { Instagram apenas uma } \\
\text { vez por dia }\end{array}$ & 0 & 3 & 3 & 13 & 19 & 9 & 47 \\
\hline Outros & 0 & 1 & 4 & 10 & 11 & 6 & 32 \\
\hline Total & 1 & 5 & 18 & 87 & 58 & 33 & 202 \\
\hline
\end{tabular}

Fonte: dados da pesquisa

Ao analisar os resultados da escala, constata-se que a maior parte dos respondentes não tem o sentimento de inveja predominante, embora poucos pesquisados tenham a tendência, segundo os números na Tabela 2. Com isso, complementa-se a compreensão do objetivo do estudo, pois é evidente que o sentimento não irá influenciar de forma direta no consumo de viagens, uma vez que, a amostra estudada viaja com frequência, utiliza o Instagram com frequência e, no entanto, não sente inveja tão determinadamente.

Muito embora os autores corroborem conceituando a inveja como um sentimento doentio, que se experimenta quando é percebido o desejo pelo que o outro possui, incluindo a vontade de apropriar-se dele ou destruí-lo (MELAINE KLEIN, 1929), em contrapartida, outra corrente entende que o sentimento pode ser controlado e tratado de outras formas, como por exemplo, a inveja e seu lado bom como o de motivar a economia, o de impulsionar esforços das pessoas a fim de trabalharem mais e com qualidade (ARISTÓTELES, 1954; EPSTEIN, 2003; KANT, 1780-1997; NEU, 1980). De qualquer modo, na próxima seção inicia-se a etapa de análise multivariada.

\subsection{Validação das Escalas}

O procedimento de validação das escalas envolveu quatro atividades: (1) análise exploratória preliminar, (2) análise de correlação, (3) análise fatorial exploratória e (4) análise de confiabilidade. A análise exploratória preliminar consistiu da avaliação visual, tratamento de missings e ouliers. O meio utilizado para coleta dos dados facilitou para que não houvesse missings. Os outliers foram considerados como observações extraordinárias inexplicáveis por se tratar de percepções subjetivas dos respondentes (HAIR et al., 2009). Dessa forma, optou-se por mantê-los, pois a análise individual destes não se apresentou como displicência ou omissão.

A terceira etapa foi realizada mediante a observação dos seguintes critérios (HAIR et al., 2009): cargas fatoriais acima de 0,50; comunalidades maiores que 0,4; variância explicada 

produtos turísticos divulgado no Instagram. Revista Hospitalidade. São Paulo, volume 15, n.02, p. 60-82, dezembro de 2018. Doi: https://doi.org/10.21714/2179-9164.2018v15n2.004

acima de 50\%; Kaiser-Meyer-Olkim (KMO) acima de 0,50 significativo ao nível p<0,05; e. Na quarta e última etapa, um Alpha de Cronbach superior a 0,60.

Tabela 4 - Indicadores Estatísticos de Validação das Escalas de Identificação Social e Inveja

\begin{tabular}{lrrccc}
\hline $\begin{array}{l}\text { Construtos e } \\
\text { Variáveis }\end{array}$ & Carga Fatorial & Comunalidades & $\begin{array}{c}\text { Variância } \\
\text { Explicada (\%) }\end{array}$ & KMO & $\begin{array}{c}\text { Alfa de } \\
\text { Cronbach }\end{array}$ \\
\hline IS1 & 0,839 & 0,704 & & & \\
IS2 & 0,929 & 0,863 & 79,58 & 0,698 & 0,867 \\
IS3 & 0,906 & 0,821 & & & \\
Inv1_Mat & 0,734 & 0,538 & & & \\
Inv5_Mat & 0,707 & 0,500 & 56,34 & 0,619 & 0,592 \\
Inv6_Mat & 0,808 & 0,652 & & & \\
Inv2_Imat & 0,733 & 0,537 & & & \\
Inv3_Imat & 0,769 & 0,591 & 56,05 & 0,640 & 0,605 \\
Inv8_Imat & 0,744 & 0,553 & & & \\
\hline
\end{tabular}

Fonte: dados da pesquisa

$\mathrm{Na}$ Tabela 4 são apresentados os indicadores. A análise fatorial exploratória para o construto Inveja retornou três fatores. As variáveis Inv1, Inv5 e Inv6 agruparam-se em um fator que os autores optaram por nomear de Inveja Material (InvMat). O fator formado pelas variáveis Inv2, Inv3 e Inv8 deu origem ao construto Inveja Imaterial (InvImat). As variáveis Inv4 e Inv5 se agruparam em um terceiro fator, mas foram retiradas da análise porque não apresentaram correlação significante.

Ressalta-se que esse procedimento foi necessário, pois nos estudos de Belk (1984, 1985), a escala não foi submetida à análise fatorial. Outro ponto que merece destaque é que a escala de Inveja utilizada nesta pesquisa tem sua gênese ligada aos estudos sobre Materialismo, o que justifica os nomes dados aos construtos.

\subsection{A Análise Fatorial Confirmatória e o Modelo de Mensuração}

Os objetivos da AFC é verificar a estrutura fatorial proposta e explorar se quaisquer modificações significativas são necessárias. Ao rodar o modelo, verificou-se que os valores agregados da AFC retornaram índices conforme o recomendado pela literatura. $\mathrm{O} \chi^{2} / \mathrm{gl}<3$, nível de significância de 0,99, RMSEA < 0,08, o IFI, o GFI e o CFI acima 0,90 (HAIR et al., 2009). 
Tabela 5 - Índices de Ajustamento Estatístico do Modelo de Mensuração

\begin{tabular}{c|c|c|c|c|c|c|c}
\hline Qui-quadrado $\left(\chi^{2}\right)$ & Graus de liberdade $(\mathrm{gl})$ & $\chi^{2} / \mathrm{gl}$ & $\mathrm{p}$ & IFI & GFI & CFI & RMSEA \\
\hline 65,225 & 36 & 1,802 & 0,002 & 0,919 & 0,925 & 0,915 & 0,077 \\
\hline
\end{tabular}

Fonte: dados da pesquisa

$\mathrm{Na}$ Figura 2, verifica-se o modelo estrutural após os ajustes indicados nos procedimentos estatísticos.

\subsection{O Modelo Estrutural e o Teste de Hipóteses}

A Modelagem de Equações Estruturais é um método estatístico utilizado para alcançar um quadro confirmatório, por meio do teste de hipóteses, da análise estrutural da teoria e de seus fenômenos. Essas teorias, em geral, representam relações causais geradas pela observação de múltiplas variáveis (BENTLER, 1988 apud BYRNE, 2010). Essa técnica veicula dois aspectos importantes de procedimento: (a) os processos causais sob estudo são representados por uma série de equações (regressões) estruturais, e (b) essas relações estruturais podem ser modeladas graficamente para clarificar a concepção da teoria sob estudo (BYRNE, 2010).

O modelo hipotético pode ser testado estatisticamente em análises simultâneas entre todas as variáveis para determinar a consistência dos dados. Em termos de indicadores, para aceitação das hipóteses, o Critical Ratio (C.R.), deve ser superior a 1,64 para $\mathrm{p} \leq 0,10$, superior a 1,96 para $\mathrm{p} \leq 0,05$, ambos para aceitação tolerável, e idealmente, deve ser superior a 2,58 para atender significância adequada de $\mathrm{p} \leq$ 0,01 (HAIR et al., 2009; BYRNE; 2010).

Das nove hipóteses testadas, quatro foram validadas, conforme Tabela 6 . Todas apresentaram o Critical Ratio (CR) negativo, sugerindo relação inversa. A frequência de uso de instagram (FI) apresentou relação significante com a Inveja Imaterial (InvImat) ao nível de 0,05 (H2b). Na prática implica afirmar que a frequência de uso do instagram pode ser indutora da inveja imaterial, ou seja, o tipo de inveja que não leva em consideração a aquisição de bens materiais, estando mais associada a aspectos intangíveis. 
SOUZA, E. M.; MONTENEGRO, R.M.B.; QUEZADO, I.; ARRUDA, D.M.O. O sentimento de inveja suscitado pelo consumo de produtos turísticos divulgado no Instagram. Revista Hospitalidade. São Paulo, volume 15, n.02, p. 60-82, dezembro de 2018. Doi: https://doi.org/10.21714/2179-9164.2018v15n2.004

Figura 2 - Modelo Estrutural Reespecificado

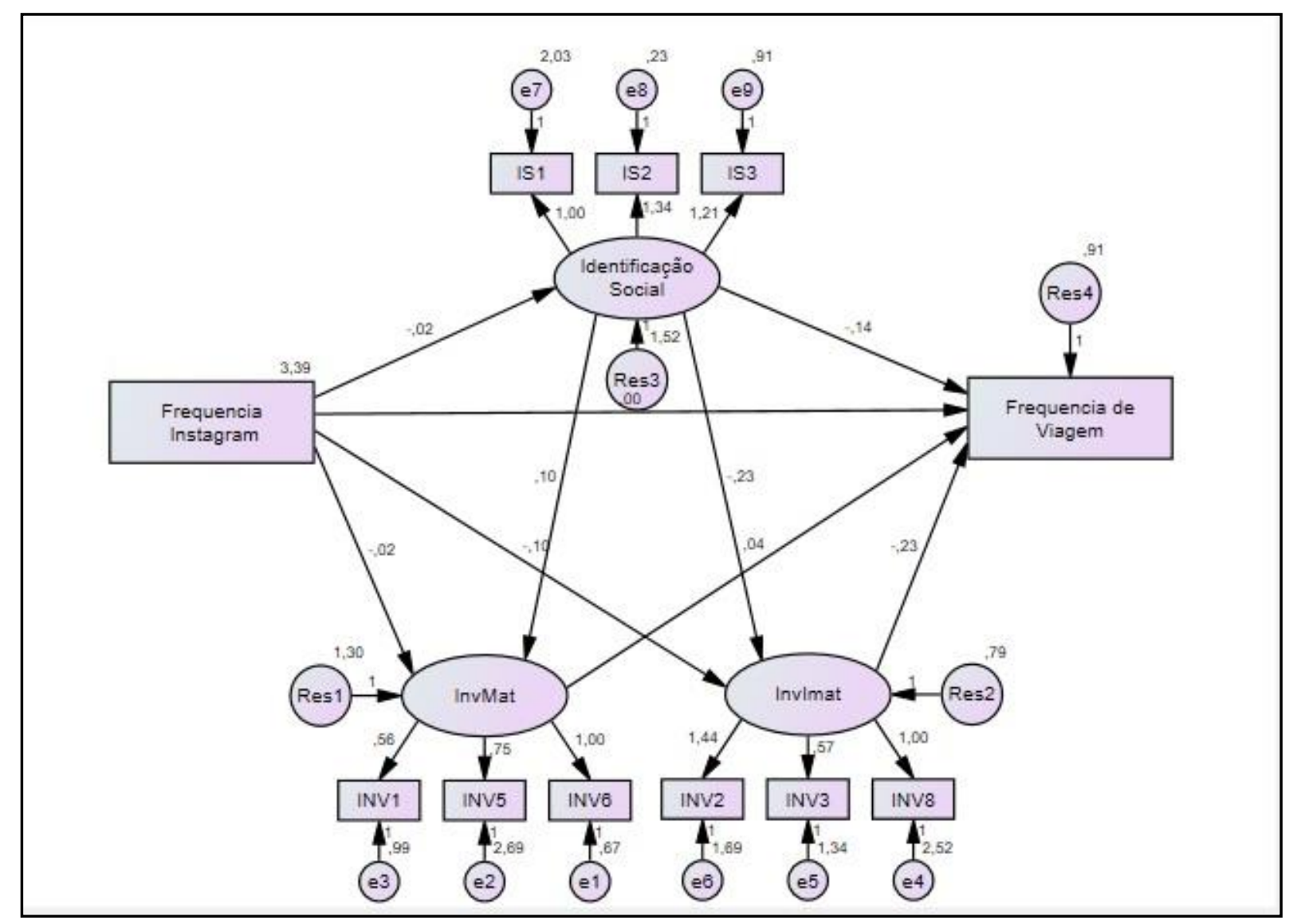

Fonte: Dados da pesquisa a partir do AMOS v. 21.

Na segunda hipótese validada (H4b), a identificação social (IS) apresentou relação significante com a inveja imaterial ao nível de 0,01. O fato do indivíduo se identificar socialmente com o público que gosta de viajar pode suscitar o sentimento de inveja imaterial nos indivíduos. 
Tabela 6 - Teste de Hipóteses para as Relações Diretas

\begin{tabular}{|c|c|c|c|c|c|c|c|c|}
\hline $\begin{array}{l}\text { Variável } \\
\text { Independente }\end{array}$ & & $\begin{array}{l}\text { Variável } \\
\text { Dependente }\end{array}$ & Estimado & S.E. & C.R. & $\mathbf{P}$ & Hipótese & Status \\
\hline FI & $\rightarrow$ & IS & $-0,025$ & 0,049 & $-0,504$ & 0,614 & H1 & $\mathrm{R}$ \\
\hline FI & $\rightarrow$ & InvMat & $-0,019$ & 0,051 & $-0,350$ & 0,726 & $\mathrm{H} 2 \mathrm{a}$ & $\mathrm{R}$ \\
\hline FI & $\rightarrow$ & Inv Imat & $-0,096$ & 0,048 & $-2,025$ & $0,046^{* * *}$ & H2b & $\mathbf{A}$ \\
\hline FI & $\rightarrow$ & $\mathrm{FV}$ & 0,001 & 0,038 & 0,024 & 0,981 & $\mathrm{H} 3$ & $\mathrm{R}$ \\
\hline IS & $\rightarrow$ & InvMat & 0,100 & 0,080 & 1,251 & 0,217 & $\mathrm{H} 4 \mathrm{a}$ & $\mathrm{R}$ \\
\hline IS & $\rightarrow$ & InvImat & $-0,229$ & 0,082 & $-2,794$ & $0,004^{* * * *}$ & H4b & $\mathbf{A}$ \\
\hline IS & $\rightarrow$ & FV & $-0,141$ & 0,064 & $-2,204$ & $0,029^{* *}$ & H5 & $\mathbf{A}$ \\
\hline InvMat & $\rightarrow$ & $\mathrm{FV}$ & $-0,036$ & 0,070 & 0,516 & 0,608 & H6a & $\mathrm{R}$ \\
\hline InvImat & $\rightarrow$ & FV & $-0,230$ & 0,106 & $-2,174$ & $0,029^{* *}$ & H6b & $\mathbf{A}$ \\
\hline
\end{tabular}

Notas: ${ }^{* *} \mathrm{p} \leq 0,05 ;{ }^{* * *} \mathrm{p} \leq 0,01 ; \mathrm{A}=$ Hipótese Aceita; $\mathrm{R}=$ Hipótese Rejeitada.

Fonte: dados da pesquisa.

A terceira hipótese validada (H5) avalia a influência da identificação social (IS) na frequência de viagens, apresentando relação significativa ao nível de 0,05. Indivíduos que são identificados socialmente tendem a intensificar sua frequência de viagens.

Por fim, ao avaliar a relação entre inveja imaterial e a frequência de viagens, encontrouse relação significativa ao nível de 0,05 . Portanto, a aquisição do sentimento de inveja imaterial influência na frequência de viagens.

A próxima etapa da análise consiste no teste das hipóteses $\mathrm{H} 7$ e $\mathrm{H} 8$, lembrando que a H8 foi dividida em H8a, H8b, H8c e H8d, em virtude da divisão do construto Inveja em Inveja Material (InvMat) e Inveja Imaterial, já justificado na análise fatorial exploratória (AFE) (TAB 7).

Para validar estas hipóteses será utilizada a mediação. Para Abbad e Torres (2002, p.21): “o conceito de mediação implica suposição de relacionamentos entre as variáveis envolvidas. Uma variável mediadora é aquela que, ao estar presente na equação de regressão, diminui a magnitude do relacionamento entre uma variável independente e uma variável dependente".

Tabela 7 - Efeito Mediador da Identificação Social e das Invejas Material e Imaterial

\begin{tabular}{|c|c|c|c|c|c|}
\hline Relação & $\begin{array}{l}\text { Efeito Direto sem } \\
\text { Mediação }\end{array}$ & $\begin{array}{l}\text { Efeito Direto com } \\
\text { Mediação }\end{array}$ & Efeito Indireto & Hipóteses & $\overline{\text { Status }}$ \\
\hline
\end{tabular}




\begin{tabular}{|c|c|c|c|c|c|}
\hline $\mathrm{FI}-\mathrm{IS}-\mathrm{FV}$ & $0,018(\mathrm{NS})$ & $0,002(\mathrm{NS})$ & $\begin{array}{r}0,462(\mathrm{NS}, \text { sem } \\
\text { mediação) }\end{array}$ & $\mathrm{H} 7$ & $\overline{\mathrm{R}}$ \\
\hline FI - InvMat - FV & $0,018(\mathrm{NS})$ & $0,002(\mathrm{NS})$ & $\begin{array}{r}0,752(\mathrm{NS}, \text { sem } \\
\text { mediação) }\end{array}$ & $\mathrm{H} 8 \mathrm{a}$ & $\mathrm{R}$ \\
\hline FI - InvImat - FV & 0,018 (NS) & $0,002(\mathrm{NS})$ & $\begin{array}{r}0,068 \text { (mediação } \\
\text { parcial) }\end{array}$ & H8b & $\mathbf{A}$ \\
\hline IS - InvMat - FV & $-0,172(0,032)$ & $-0,108(0,140)$ & $\begin{array}{r}0,543 \text { (NS, sem } \\
\text { mediação) }\end{array}$ & $\mathrm{H} 8 \mathrm{c}$ & $\mathrm{R}$ \\
\hline IS - InvImat - FV & $-0,147(0,027)$ & $0,177(0,029)$ & $\begin{array}{r}0,019 \text { (mediação } \\
\text { completa) }\end{array}$ & H8d & $\mathbf{A}$ \\
\hline
\end{tabular}

Fonte: dados da pesquisa a partir do AMOS v 21.

Um dos pré-requisitos para o uso da mediação é que haja relação significante entre as variáveis dependente e independente, como se trata de uma análise exploratória, optou-se por testar estatisticamente as diversas relações possíveis conforme modelo apresentado na Figura 1 e ajusta na Figura 2. Aplicou-se bootstraping com 5.000 reamostras, um dos pressupostos para esse tipo de análise (VIEIRA, 2009).

O primeiro passo foi verificar o Efeito Direto sem Mediação, para tal utilizou-se os valores das Cargas Padronizadas e a existência de significância da relação, apresentadas na Tabela 7, respectivamente. Da mesma forma, foi realizado o teste com a inserção das variáveis moderadoras, neste caso - IS, InvMat e InvImat - analisando assim, se o uso da variável moderadora enfraquece a relação direta, o que caracteriza a mediação. Em seguida, verificou-se se o efeito direto foi significativo, apresentando $p$-value igual ou menor a 0,01 .

Das cinco hipóteses testadas nessa etapa, apenas a H8b e H8d foram validadas. Na hipótese H8b foi testado se a Inveja Imaterial (InvImat) media a relação entre a frequência de uso do Instagram (FI) e a Frequência de Viagens. Segundo Vieira (2009), encontrou-se uma mediação parcial, que ocorre quando a relação entre a variável mediadora, nesse caso a Inveja Imaterial, e a variável dependente (FI) e independente (FV) são não significantes (NS). Na hipótese H8d foi testado se a Inveja Imaterial (InvImat) media a relação entre Identificação Social (IS) e Frequência de Viagens (FV). Para essa hipótese os testes demonstraram haver mediação parcial.

Das três variáveis testadas como mediadoras, apenas a Inveja Imaterial (InvImat) mostrou-se significativa, mediando as duas relações propostas, indicando assim, necessidade de se ampliar e aprofundar o conceito e o entendimento da Inveja Imaterial.

\section{CONCLUSÃO}


Este trabalho teve como objetivo verificar o papel do sentimento de inveja no consumo de viagens a partir do conteúdo visualizado no Instagram. Os resultados indicaram que a amostra foi formada majoritariamente por pessoas que possuem identificação social relacionada ao consumo de viagens e se mostraram não propensas ao sentimento de inveja.

Das 14 hipóteses testadas, 6 foram validadas. Destaque-se o construto Inveja Imaterial que esteve presente em 5 das 6 hipóteses confirmadas, sendo, portanto, o construto mais relevante dessa investigação. A única hipótese confirmada que não envolveu a InvImat foi a H5, na qual foi testada se a Identificação Social (IS) influencia a Frequência de Viagens.

Embora não se tenha tecido comentários sobre as hipóteses rejeitadas, até porque essa não era a proposta, essas hipóteses deixam como contribuição o "que não fazer" nas próximas pesquisas que busquem ampliar a compreensão do sentimento de inveja suscitado nas relações de consumo.

Os construtos propostos nessa pesquisa não são de uso comum nas pesquisas de marketing, mais especificamente relativas à comportamento do consumidor. Encontrar e adaptar escalas para essa pesquisa foi o primeiro grande desafio encontrado para empreender essa pesquisa. Outra particularidade dessa pesquisa foi a delimitação do campo empírico, as mídias sociais, mais especificamente o Instagram, cujo comportamento diferem dos encontrados em um ambiente real. Dadas essas particularidades, o estudo empreendido foi eminentemente exploratório.

Além destas características, o estudo contribui para o setor turístico que vem se destacando no mercado consideravelmente. Porém, a pesquisa teve limitações que merecem registros. A primeira delas foi em relação a investigação do sentimento de inveja dos pesquisados. Mesmo destacando que fosse necessário o máximo de sinceridade nas respostas, não se pode proferir com exatidão que houve a total verdade.

Como sugestão de estudos futuros, recomenda-se verificar o sentimento por meio de outras redes sociais como o Facebook, Twitter e em outros nichos de mercado relevantes. Metodologicamente, inclusão de novos construtos ao modelo teórico, como por exemplo, uso de escalas para consumo no meio virtual. Indica-se, ainda, o uso de pesquisa qualitativa, podendo esta contribuir para a construção de escalas mais atualizadas e que representem o contexto de consumo contemporâneo. 


\section{REFERÊNCIAS}

ARISTOTELES. The Rhetoric and the Poetics of Aristotle. ed. W. Rhys Roberts. New York: Modern Library, 1954.

BABBIE, E. Métodos de Pesquisas de Survey. Tradução: Guilherme Cezarino. Belo Horizonte: Ed. UFMG, 2001.

BAUDRILLARD, J. A sociedade de consumo. Lisboa: Edições 70, 2005.

BELK, R. W. Three Scales to Measure Constructs Related to Materialism: Reliability, Validity, and Relationships to Measures of Happiness. Advances in Consumer Research. Ohio, EUA. vol. 11. p. 291-297, 1984.

BELK, R. W. Materialism: Trait Aspects of Living in the Material World. Journal of Consumer Research. Ohio, EUA. vol. 12. p. 265-280, 1985.

BOYD, D. M.; ELLISON, N. B. Social network sites: definition, history, and scholarship. Journal of Computer-Mediated Communication, vol. 13, Vol. 1, artigo 11, 2007.

DESCARTES, R. As paixões da alma. Ed. 2. São Paulo: Abril Cultural Editora, 1979. 324 p.

CORRAR, L. J. et al. Análise Multivariada: para os cursos de Administração, Ciências Contábeis e Economia. São Paulo: Ed. Atlas, 2012.COSTA, F. J. Mensuração e Desenvolvimento de Escalas: Aplicações em Administração. Rio de Janeiro: Editora Ciência Moderna, 2011.

EPSTEIN, J. Envy: The Seven Deadly Sins. New York: Oxford University Press, 2003. 144 p.

ESCALAS, J. E.; BETTMAN, J. Self-Construal, Reference Groups, and Brand Meaning, JCR, 32 (December), p. 378-389, 2005.

FÁVERO, L. P.; BELFIORE, P.; SILVA, F. L.; CHAN, B. L. Análise de dados: modelagem multivariada para tomada de decisões. Rio de Janeiro: Elsevier, 2009.

FIALHO, C. B.; LUTZ, C. B. Análise da intenção de continuidade de uso de um sistema voluntário: Em cena o fenômeno twitter. In: Encontro da Administração da Informação III. 2011, Porto Alegre, RS.

FREUD, S. Group psychology and the analysis of the ego. Journal of Nervous and Mental Disease. Baltimore, USA. vol. 60, p. 434-436, 1924.

HAIR, J. F.; BLACK, W. C.; BABIN; B. J.; TATHAM; R. L. Análise Multivariada de Dados. $6^{\mathrm{a}}$ ed. Porto Alegre: Bookman, 2009. 
KANT, Immanuel. "Lectures on Ethics," In: The Cambridge Edition of the Works of Immanuel Kant, ed. PeterHeath and Jerome B. Schneewind. Cambridge: Cambridge University Press, 1780-1997.

KLEIN, Melaine. Personification in the play of children. The International Journal of Psychoanalysis. USA, 1929.

MACHADO, D. F. C.; PIMENTEL, T. D. Os Reflexos da Teoria do Comportamento do Consumidor nos Estudos sobre a Imagem do Destino Turístico. Anais... Encontro de Marketing da Anpad III (EMA), Curitiba, 2008.

MARÔCO, J. Análise de equações estruturais: fundamentos teóricos, software \& aplicações. Pêro Pinheiro: ReportNumber, 2010.

MORAES, G. H. S. M.; CAPELLOZZA, A.; MEIRELLES, F. S. \#VEMPRARUA: A Tecnologia da Informação e as Manifestações Sociais: Um Estudo da Utilização das Redes Sociais para Participação nos Protestos. Anais... Encontro da Anpad XXXVIII (Enanpad), Rio de Janeiro, 2014.

NEU, J. Jealous Thoughts In: Explaining Emotions, ed. Amelie O. Rorty. Los Angeles: University of California. p. 425-463. 1980.

RAACKE, J.; BONDS-RAACKE, J. Myspace and facebook: Applying the uses and gratifications theory to exploring friend-networking sites. CyberPsychology \& Behavior, v. 11, n. 2, p. 169-174, 2008.

RECUERO, R. Redes sociais na Internet. Porto Alegre: Editora Meridional, 2009. 191 p.

ROSEMAN, I. J.; CYNTHIA, W.; TAMARA, S. S. Phenomenology, Behaviors, and Goals Differentiate Discrete Emotions. Journal of Personality and Social Psychology. vol. 67, p. 206-221, 1994.

SAMPAIO, C.A.C.; GANDARA, J. M. G.; MANTOVANELLI JR, O. Turismo como fenômeno humano: uma nova base conceitual para implementação de políticas públicas. In: V Seminário de Pesquisa em Turismo do MERCOSUL (SeminTUR- 2008 - Caxias do Sul). Anais Eletrônicos, Rio Grande do Sul, 2008. Disponível em: http://www.ucs.br/ucs/tplVSeminTur > acesso em: março/2015

SMITH, R. H.; KIM. S. H. Comprehending Envy. Psychological Bulletin, v.13, n.1, p. 46-64, 2007.

SCHOECK, H. Envy: A Theory of Social Behavior. New York: Harcourt, Brace \& World, 1969.

TOMEI, P. A.; BELLE, F. Análise Comparativa da Gerência da Inveja nas Organizações Brasileiras e Francesas. Revista da RAUSP. São Paulo. v. 32. n. 1. p. 5-13, 1997. 
SOUZA, E. M.; MONTENEGRO, R.M.B.; QUEZADO, I.; ARRUDA, D.M.O. O sentimento de inveja suscitado pelo consumo de produtos turísticos divulgado no Instagram. Revista Hospitalidade. São Paulo, volume 15, n.02, p. 60-82, dezembro de 2018. Doi: https://doi.org/10.21714/2179-9164.2018v15n2.004

VAN DE VEN, Niels; ZEELENBERG, Marcel; PIETERS, Rik. Leveling Up and Down: The Experience of Benign and Malicious Envy. Emotion, v. 9. p. 419-429, 2009.

Artigo recebido em: 09/09/2018

Avaliado em: 10/09/2018

Aprovado em: 10/09/2018 